\title{
Abnormal eating attitudes and weight-loss behaviour of adolescent girls attending a "traditional" Jewish high school in Johannesburg, South Africa
}

\author{
Visser J, BSc Dietetics, M Nutrition; Senior Lecture \\ Division of Human Nutrition, Faculty of Medicine and Health Sciences, Stellenbosch University; Tygerberg Academic Hospital \\ Notelovitz T, MBBCh, MNutrition; Private Practice \\ Division of Human Nutrition, Faculty of Medicine and Health Sciences, Stellenbosch University \\ Szabo CP, MBBCh, FCPsych, MMed, PhD, MSc(Med), Professor and Head \\ Department of Psychiatry, Faculty of Health Sciences, University of the Witwatersrand, Johannesburg \\ Fredericks N, ${ }^{1} \mathrm{BSc}$ (Dietetics), MSc(Nutrition Management) \\ Division of Human Nutrition, Faculty of Medicine and Health Sciences, Stellenbosch University; Chief Dietitian, Tygerberg Hospital \\ Correspondence to: Janicke Visser, e-mail: jconrad@sun.ac.za \\ Keywords: eating attitudes, weight-loss behaviour, Jewish, adolescents, high school, Johannesburg
}

\section{Abstract}

Objectives: This study aimed to determine the prevalence of abnormal eating attitudes and weight-loss behaviour in female Jewish adolescents. Teachers' awareness of these factors and their attitudes towards a school programme to address these were also investigated.

Design: A cross-sectional study was conducted.

Subjects and setting: Female learners in grades 8-11 $(\mathrm{n}=220)$, attending a "traditional" Jewish high school in Johannesburg were included. Teachers $(n=38)$ at the relevant school were also recruited.

Outcome measures: A questionnaire consisting of the 26-item version of the Eating Attitudes Test (EAT-26) and a modified section of the USA Youth Risk Behavior Survey was completed by learners. Teachers completed a questionnaire designed by the researchers.

Results: Twenty per cent of the learners $(n=43)$ achieved EAT-26 scores $\geq 20$, suggestive of a possible eating disorder, while $30.2 \%$ $(n=65)$ required clinical evaluation for a potential eating disorder. Thirty-three per cent $(n=72)$ of the adolescent girls considered themselves to be overweight, while $64 \%(n=139)$ were trying to lose weight at the time of the study. $19.1 \%(n=42)$ had engaged in one or more extreme methods of weight loss in the past 12 months. Most teachers $(81.6 \%, n=29)$ underestimated the proportion of adolescent girls requiring clinical evaluation and $71.1 \%(n=27)$ underestimated the extent of current weight-loss attempts. Almost all of the teachers $(97.3 \%, n=37)$ recognised the need to address disordered eating attitudes. However, only $34.2 \%$ of the teachers $(n=13)$ viewed the school as the appropriate place in which to do this, and were also prepared to participate in the programme and sacrifice class time.

Conclusion: To date, no published South African literature documents the presence of abnormal eating attitudes in Jewish adolescent females in South Africa. The prevalence fell within the upper end of rates reported in studies on adolescent girls in South Africa and abroad. Teachers who participated in this study were not fully aware of the extent to which eating-related issues affected female learners. A qualitative exploration thereof could yield valuable insights.

\section{Introduction}

Eating disorders have been characterised as being "of great interest to the public, of perplexity to researchers, and a challenge to clinicians". ${ }^{1}$ They are believed to have increased in prevalence in recent decades, and it is postulated that they relate to increasing socio-cultural pressure to be thin. ${ }^{1,2}$ Adolescent and young adult females are most at risk. ${ }^{3}$ Eating disturbances in this population are the third most common chronic condition in adolescent girls in the USA, after obesity and asthma. ${ }^{4}$ Eating disorders can be classified as anorexia nervosa, bulimia nervosa and eating disorder not otherwise specified, and can result in a multitude of life-threatening conditions. ${ }^{1,5}$

No direct causal relationship has been found to explain the development of eating disorders, although available data support the notion that interplay exists between environmental and genetic factors. Ethnocultural influences, the effect of media exposure and a changing social and cultural environment are contributing factors to the aetiology of eating disorders. ${ }^{2,6-8}$ The relationship between religion and eating disorders is also not to be underestimated, given its complexity. .10

The existence of eating disorders and abnormal eating attitudes in South Africa are well established in adolescents of culturally and ethnically diverse backgrounds, ${ }^{11-15}$ although the prevalence thereof is currently unknown. ${ }^{16}$ International studies have found disturbances of this nature to be more prevalent in Jewish adolescent girls and women than in their non-Jewish counterparts. ${ }^{17-19}$

The emergence of data suggesting that Jewish girls and women may be at an increased risk of developing eating disorders ${ }^{17-19}$ 
is of concern to the global Jewish community. Food is often seen to be a focal point of Jewish traditions, spanning cultural, ethnic and religious paradigms. ${ }^{20}$ The prevalence of abnormal eating attitudes in Johannesburg Jewish high school girls is currently unknown, and the determination thereof could have important implications for the implementation of preventative strategies. Drawing attention to the existence of disturbed eating patterns could promote earlier identification and referral for treatment of clinical or subclinical eating disturbances by parents and teachers. Prior research suggests that eating attitudes in Jewish adolescents may vary in learners with different levels of religious adherence. ${ }^{21-23}$ This study focused on the traditional sector of the population. According to previous research, this sector may well contain higher levels of disordered eating than those of the more orthodox groups. ${ }^{21-23}$ However, a recent study conducted in Israel showed that differing Jewish religious groups displayed similar patterns of disordered eating behaviour, despite the more arduous religious observance of the ultra-Orthodox group. ${ }^{20}$

To the authors' knowledge, there are no published studies on eating attitudes and/or eating disorders in the South African Jewish community. This was also the first study to document teachers' willingness to participate in preventative programmes. Thus, the aim of this study was to determine the prevalence of abnormal eating attitudes and weight-loss behaviour in a Jewish female adolescent sample, and to investigate teachers' awareness of these factors. Teachers' attitudes towards a school programme to address these factors were also investigated.

\section{Method}

\section{Study population}

This cross-sectional study evaluated two distinct sectors of the study population; female learners and teachers of both genders at a private "traditional" Jewish high school in Johannesburg. Initially, the aim was to include girls from grades $8-12$, but owing to logistical complications, the grade 12 girls were unable to participate. Therefore, the total number of available adolescent girls for the study was 261 , in grades $8-11$ ( $77.2 \%$ of the girls in the school). Eligible adolescent girls were included as current learners and teachers were considered to be a statistically sound sample of those attending or teaching at the school.

In addition, participants were also included if they were selfidentified followers of the Jewish faith, provided that both their own and parental or guardian written consent had been given, that they displayed good comprehension of the English language and were present at school on the day that the questionnaire was administered.

\section{Questionnaires}

Two written, self-administered English questionnaires were used for the learners and the teachers, respectively. The learners' questionnaire consisted of three sections; demographic data, the 26-item version of the Eating Attitudes Test (EAT-26), ${ }^{24}$ and selected questions from the USA Youth Risk Behavior Survey (YRBS). ${ }^{25}$

The EAT-26 is a free-of-charge, 26-item, self-report questionnaire with three subscales of bulimia, dietary restraint and oral control or food preoccupation. A score of 0-3 is given for each item, and the total score determined by adding each individual score. Although the test is not intended to be diagnostic of an eating disorder, a score of $\geq 20$ was suggestive of a possible eating disorder, requiring further investigation. ${ }^{24}$ The EAT-26 has been successfully used to identify individuals with disturbed eating patterns and attitudes in non-clinical samples, including populations of Jewish high school adolescent girls in Israel ${ }^{22,23}$ and Canada, ${ }^{19}$ and South African high school girls of varying ethnicities. 12,14,26,27

Questions dealing with weight-loss behaviour were extracted from the most recent YRBS, ${ }^{25}$ which deals with unhealthy dietary behaviour. The YRBS was designed by the Centers for Disease Control and Prevention to monitor behaviour that contributes markedly to leading causes of morbidity, mortality and social problems in USA youth..$^{28}$ Minor adaptations were made to the YRBS to suit the study population and outcomes, and included additional culture-, population- and demographic-specific questions.

The researchers developed the teachers' questionnaire in line with the aims of the study, with questions designed in parallel with those of the learners. Additional information included personal information on each teacher, as well as profession-specific questions.

A small pilot study was conducted to ensure face validity and to determine the time necessary for questionnaire completion. Four adolescent girls (one per grade), and two teachers (one male and one female), were selected for participation via convenience sampling. These individuals were later excluded from the final study. Questionnaires were collected and examined by the researcher for errors in completion.

The questionnaires were completed over a two-week period by consenting female learners during class time, and were supervised by either the researcher or the facilitating teacher. Verbal and written instructions were given to the learners beforehand. The teachers were approached by the researcher on two separate occasions during compulsory weekly staff meetings. Willing teachers also received verbal and written instructions before questionnaire completion.

\section{Ethical considerations}

Ethics approval was obtained from the Health Research Ethics Committee of the Faculty of Medicine and Health Sciences, Stellenbosch University (N09/05/154). Participation by both the learners and teachers was voluntary. The anonymity of the participants was ensured via the omission of subject identification information and the school in question was not referred to by name. Consent from the school principal, as well as the learners and their respective parents or guardians, was obtained prior to commencement of the study. Teachers were informed that completion of the questionnaire implied their consent.

\section{Data analysis}

Data were analysed using Statistica ${ }^{\circledR}$ version 9.0 and $\mathrm{SAS}^{\circledR}$ version 9.1. Questionnaires that were incompletely or incorrectly completed were excluded only from analyses relating to the particular question. The total score for the EAT-26 was not calculated for participants 
who had omitted or completed one or more items incorrectly. Binary terms were created on the basis of the recommended cut-off of an EAT-26 score of $\geq 20$ as an indication of a possible eating disorder that required evaluation for each of the items relating to weight-loss behaviour in the past year, and for each of the questions relating to the teachers' attitudes. T-tests were used to compare means. Best subsets regression was utilised to determine the best possible predictors of total EAT-26 score, EAT-26 score $\geq 20$, and the size and accuracy of teachers' predictions. Methods of best subset regression include adjusted R-squared, Mallows' Cp and forward stepwise regression. Odds ratios were calculated for the best predictors identified in the regression analysis. The analyses were examined for significance at the $5 \%$ level.

\section{Results}

The total available population of female learners comprised 261 adolescent girls in grades 8-11 (Figure 1). The final learner population included in this study was 220 adolescent girls (84.3\%).

Twenty-six per cent $(n=56)$ of the participants were in grade 8 , $24.5 \%(n=54)$ in grade $9,23.2 \%(n=51)$ in grade 10 and $26.8 \%(n$ $=59$ ) in grade 11 . There was no significant difference between the response rates in the different grades ( $p$-value 0.67). Participants' ages ranged from 13.58-18.25 years, with a mean of 15.68 [standard deviation (SD) 1.17].

\section{Eating Attitudes Test (26-item version) scores}

The EAT-26 scores for the participants ranged from 0-51, with a mean of 12.1 (SD 11.11). Correlation was not found between the total EAT-26 score and age ( $p$-value 0.35). The total EAT-26 score was significantly associated with the grade ( $p$-value 0.04 ), as there were lower average scores for grades 8 and 10 than for grades 9 and 11.

Twenty per cent of the participants $(n=43)$ scored $\geq 20$ on the EAT-26, which is suggestive of a possible eating disorder. The association between the grade and having a score of $\geq 20$ was significant ( $p$-value 0.03 ), although this was not found to be a

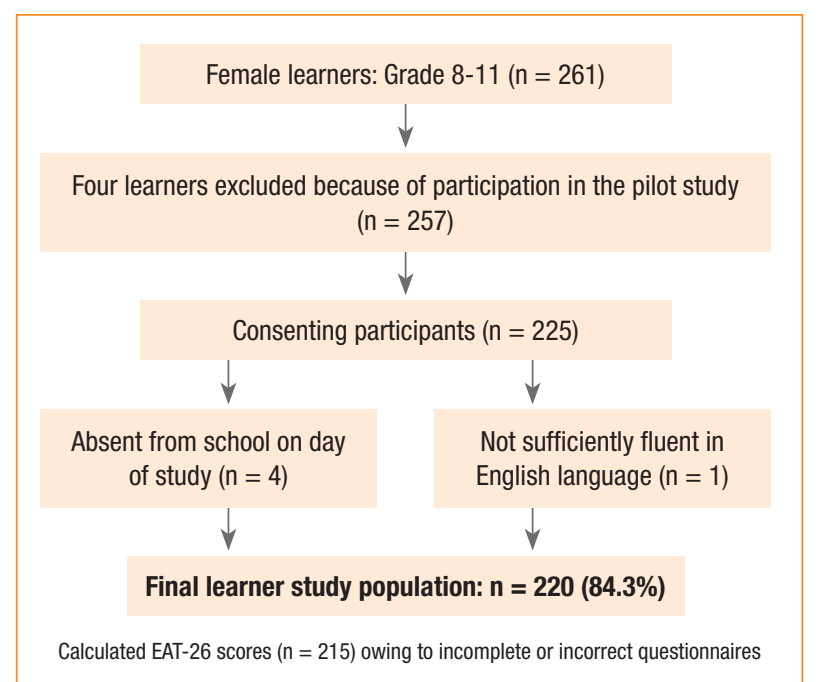

EAT-26: Eating Attitudes Test (26-item version)

Figure 1: Final learner study population significant predictor of an EAT-26 score $\geq 20$. Age was not found to be significantly associated with having an EAT-26 score of more than or equal to 20 ( $p$-value 0.39 ).

\section{Scores for individual items on the Eating Attitudes Test (26-item version)}

The item which most frequently received a score of above zero was item 12 , followed by item 26 , and lastly item 1 (Table I). Only $5 \%$ $(n=11)$ of adolescent girls in the entire group responded with "never" to item 1 . The lowest percentage of learners $(0.9 \%, n=2)$ who scored above zero pertained to item 9.

The item with the highest mean score was item 12 (mean score 1.37, SD 1.24) followed by item 1 (mean score 1.31, SD 1.28). The six items with the highest average scores are marked with an asterisk in Table I:

- "I am terrified of being overweight."

- "I am preoccupied with a desire to be thinner."

- "I think about burning up calories when I exercise."

- "I am preoccupied with the thought of having fat on my body."

- "I display self-control around food."

- "I enjoy trying rich new foods".

The lowest mean score pertained to item 9 (mean score 0.02 , SD 0.19).

\section{Predictors of total Eating Attitudes Test (26-item version) scores}

The following predictors were selected as the best possible indicators of a total EAT-26 score $\geq 20$ : adolescent girls who regarded themselves as being overweight (either slightly or very overweight) ( $p$-value 0.00 ), those who had dieted in the past year ( $p$-value 0.03 ), and whether or not extreme methods of achieving weight-loss had been employed ( $p$-value 0.00 ). Surprisingly, currently trying to lose weight was not selected, possibly because the finding was so common in the study population.

Adolescent girls who viewed themselves as being overweight displayed a 3.47 times greater likelihood of having an EAT-26 score of $\geq 20$, whereas those who had dieted or used extreme weight-loss were 3.21 and 3.91 , respectively, more likely to have EAT-26 scores $\geq 20$.

\section{Weight management goals and self-perceived weight}

Two hundred and fourteen (99.5\%) of the participants completed the questions on weight management goals and self-perceived weight. Sixty per cent $(n=129)$ viewed themselves as being the correct weight, $30.4 \%(n=65)$ to be slightly overweight, $3.3 \%(n=7)$ to be very overweight, and $6.1 \%(n=13)$ to be slightly underweight (Figure 2).

Sixty four per cent $(n=137)$ of the participants were trying to lose weight at the time of the study, $21.5 \%(n=46)$ wished to maintain their current weight, $13.6 \%(n=29)$ were not trying to achieve anything with their weight, and $0.9 \%(n=2)$ were actively trying to gain weight.

Of the adolescent girls who viewed themselves as being the correct weight, the majority (56.6\%) were attempting to lose weight at the time that the study was conducted. Of the 13 girls who regarded 
Table I: Mean score for each item on the Eating Attitudes Test (26-item version)

\begin{tabular}{|c|c|c|c|c|}
\hline Item & Statement & Valid (n) & Mean & SD \\
\hline $1^{*}$ & I am terrified of being overweight & 220 & 1.31 & 1.28 \\
\hline 2 & I avoid eating when I am hungry & 220 & 0.05 & 0.26 \\
\hline 3 & $\begin{array}{l}\text { I find that I am preoccupied with } \\
\text { food }\end{array}$ & 219 & 0.32 & 0.72 \\
\hline 4 & $\begin{array}{l}\text { I have gone on eating binges and felt } \\
\text { that I may not be able to stop eating }\end{array}$ & 220 & 0.20 & 0.59 \\
\hline 5 & I cut my food into small pieces & 220 & 0.24 & 0.65 \\
\hline 6 & $\begin{array}{l}\text { I am aware of the calorie content of } \\
\text { the foods that I eat }\end{array}$ & 220 & 0.55 & 0.97 \\
\hline 7 & $\begin{array}{l}\text { I particularly avoid food with a high- } \\
\text { carbohydrate content, e.g. bread, } \\
\text { rice and potatoes }\end{array}$ & 220 & 0.36 & 0.78 \\
\hline 8 & $\begin{array}{l}\text { I feel that others would prefer it if I } \\
\text { ate more }\end{array}$ & 220 & 0.15 & 0.56 \\
\hline 9 & I vomit after I have eaten & 220 & 0.02 & 0.19 \\
\hline 10 & I feel extremely guilty after eating & 219 & 0.24 & 0.66 \\
\hline $11^{*}$ & $\begin{array}{l}\text { I am preoccupied with a desire to } \\
\text { be thinner }\end{array}$ & 220 & 0.93 & 1.21 \\
\hline $12^{*}$ & $\begin{array}{l}\text { I think about burning up calories } \\
\text { when I exercise }\end{array}$ & 220 & 1.37 & 1.24 \\
\hline 13 & Other people think that I am too thin & 220 & 0.18 & 0.60 \\
\hline $14^{*}$ & $\begin{array}{l}\text { I am preoccupied with the thought of } \\
\text { having fat on my body }\end{array}$ & 220 & 0.78 & 1.11 \\
\hline 15 & $\begin{array}{l}\text { I take longer than others to eat my } \\
\text { meals }\end{array}$ & 220 & 0.38 & 0.84 \\
\hline 16 & I avoid foods with sugar in them & 220 & 0.17 & 0.58 \\
\hline 17 & I eat diet foods & 219 & 0.59 & 0.97 \\
\hline 18 & I feel that food controls my life & 219 & 0.36 & 0.88 \\
\hline $19^{*}$ & I display self-control around food & 220 & 0.81 & 1.07 \\
\hline 20 & I feel that others pressure me to eat & 220 & 0.08 & 0.40 \\
\hline 21 & $\begin{array}{l}\text { I give too much time and thought } \\
\text { to food }\end{array}$ & 220 & 0.43 & 0.90 \\
\hline 22 & $\begin{array}{l}\text { I feel uncomfortable after eating } \\
\text { sweets }\end{array}$ & 220 & 0.50 & 0.93 \\
\hline 23 & I engage in dieting behaviour & 220 & 0.66 & 1.03 \\
\hline 24 & I like my stomach to be empty & 220 & 0.27 & 0.76 \\
\hline 25 & $\begin{array}{l}\text { I have an impulse to vomit after } \\
\text { meals }\end{array}$ & 220 & 0.13 & 0.52 \\
\hline $26^{*}$ & I enjoy trying rich new foods & 219 & 1.08 & 1.05 \\
\hline
\end{tabular}

SD: standard deviation

Mean scores and standard deviations for these data are quoted to two decimal places to allow comparisons to be made between the means in a narrow range. The six items with the highest average scores are marked with an asterisk

themselves as being underweight, only $1(7.7 \%)$ were trying to gain weight, whereas 7 (53.9\%) were trying to remain the same weight, or were not doing anything about their weight $(23.3 \%, n=3)$. Of the adolescent girls who viewed themselves as being underweight, $15.4 \%(n=2)$ were actively trying to lose weight. The proportion of girls trying to lose weight at the time of the study was highest in the group who regarded itself as being slightly overweight $(87.7 \%$, $\mathrm{n}=57$ ) and was higher in this group than in the group who regarded itself as being very overweight $(71.4 \%, n=5)$.

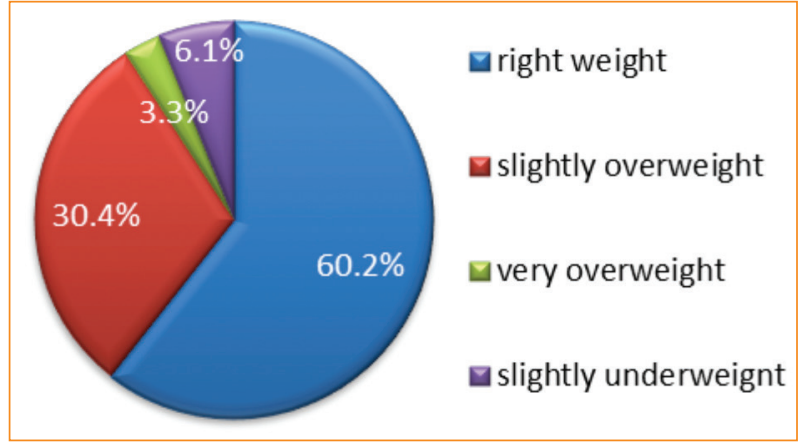

Figure 2: Categorisation of sample by self-perceived weight

\section{Weight-management strategies used within the past 12 months}

The most commonly used weight management strategy was exercise $(77.8 \%, n=171)$, followed by diet $(67.3 \%, n=148)$, fasting $(10.0 \%, n=22)$, purging $(8.6 \%, n=19)$ and non-prescribed medication $(6.8 \%, n=15)$. Nineteen per cent of the subjects ( $n=42$ ) used an extreme method of weight-loss (fasting, purging or non-prescribed medication). There were no significant differences between the grades in terms of the method of weight-loss employed. Of the group trying to lose weight at the time of the study, $87.7 \%$ $(n=122)$ had exercised, $81.3 \%(n=113)$ had dieted, and $24.4 \%$ $(\mathrm{n}=34$ ) had used extreme methods of weight-loss. Twenty-seven per cent $(n=38)$ of adolescent girls in this group had an EAT-26 score of $\geq 20$.

\section{Learners requiring professional evaluation}

Adolescent girls who required evaluation by a healthcare professional for a potential eating disorder were defined as either having an EAT26 score of $\geq 20$, or alternatively as having engaged in one or more of the extreme methods of weight-loss in the past year. Thirty per cent of them in the school $(n=65)$ were found to require evaluation for a potential eating disorder.

\section{Eating disorder diagnosis}

Four per cent $(n=9)$ of the sample reported having previously been diagnosed as having an eating disorder, with the difference between grades not being significant ( $p$-value 0.05 ). Of the adolescent girls who required evaluation by a healthcare professional as previously defined, only $9.2 \%(n=6)$ reported having previously been diagnosed with an eating disorder.

\section{Teachers' questionnaire}

Thirty-eight teachers (male and female) participated in the study (a $52.1 \%$ participation rate). The majority of teachers were women $(71.1 \%, n=27)$ and of the Jewish faith $(63.2 \%, n=24)$. Forty-seven per cent $(n=18)$ taught grade $8,57.9 \%(n=22)$ grade $9,76.3 \%$ $(\mathrm{n}=29)$ grade $10,63.2 \%(\mathrm{n}=24)$ grade 11 and $60.5 \%(\mathrm{n}=23)$ grade 12 . The mean age of the participants was 44.6 years (SD 14.23). The mean time spent teaching was 18 years (SD 13.57), whereas the mean time spent teaching at the specific school was 9.7 years (SD 9.66). 
Table II: Comparison between teachers' estimates and the results calculated from the learners' questionnaire

\begin{tabular}{|l|c|c|c|c|c|c|}
\hline \multirow{2}{*}{ Category } & \multicolumn{5}{|c|}{ Teachers' estimates } \\
\cline { 2 - 7 } & $\begin{array}{c}\text { Learners' questionnaire } \\
\text { results (\%) }\end{array}$ & n (valid) & Mean (\%) & SD & Range & p-values \\
\hline Diagnosed with an eating disorder & 4.1 & 35 & 11.5 & 12.53 & $2-50$ & 0.00 \\
\hline $\begin{array}{l}\text { Extreme method of weigh-loss over the past year } \\
\text { Believe that they are overweight }\end{array}$ & 19.1 & 34 & 25.9 & 19.55 & $2-70$ & 0.05 \\
\hline $\begin{array}{l}\text { Currently trying to lose weight } \\
\text { Dieted in the past year (to lose weight or prevent }\end{array}$ & 33.7 & 36 & 49.2 & 27.52 & $5-90$ & 0.00 \\
\hline weight gain) & 64 & 37 & 52.3 & 22.24 & $10-90$ & 0.01 \\
\hline $\begin{array}{l}\text { Exercised in the past year (to lose weight or prevent } \\
\text { weight gain) }\end{array}$ & 67.3 & 37 & 49.7 & 25.09 & $8-100$ & 0.00 \\
\hline Need evaluation for a potential eating disorder & 77.8 & 36 & 46.7 & 23.30 & $5-80$ & 0.00 \\
\hline
\end{tabular}

SD: standard deviation

\section{Teachers' estimates}

The mean of the teachers' estimates (predictions of learner responses) was significantly higher than the percentage calculated from the learners' self-report questionnaires for the percentage of adolescent girls who had previously been diagnosed with an eating disorder (overestimated by $76.3 \%, n=29$ of teachers) ( $p$-value 0.00 ), and those learners who believed that they were overweight (overestimated by $63.2 \%, \mathrm{n}=24$ of teachers) ( $\mathrm{p}$-value 0.00 ) (Table II).

The mean of the teachers' estimates was significantly lower than the percentage calculated from the learners' self-report questionnaire for the percentage of adolescent girls who had dieted in the past year to lose weight or to prevent weight gain (underestimated by $73.7 \%$, $\mathrm{n}=28$ of teachers) (p-value 0.00 ), those who had exercised in the past year to lose weight or prevent weight gain (underestimated by $84.2 \%, n=32$ of teachers) ( $p$-value 0.00 ), those who were trying to lose weight at the time of the study (underestimated by $71.1 \%$, $\mathrm{n}=27$ of teachers) (p-value 0.01 ), and those requiring evaluation by a healthcare professional to determine if there was a potential eating disorder (underestimated by $81.6 \%, \mathrm{n}=31$ of teachers) (p-value 0.00).

Total teaching time yielded significant results when the predictive accuracy of the teachers' estimates was assessed, as a longer teaching time gave rise to greater accuracy in predicting the following:

- Learners who regarded themselves as being overweight ( $p$-value $0.00)$.

- Those trying to lose weight at the time of the study ( $p$-value 0.00 ).

- Those who had dieted in the past year ( $p$-value 0.00$)$.

- Those who had exercised in the past year ( $p$-value 0.00 ).

- Those who had used extreme weight-loss methods in the past year ( $p$-value 0.02).

\section{Teachers' attitudes to preventative programmes}

Almost all of the teachers $(97.3 \%, n=37)$ recognised the need to address the eating attitudes of adolescent girls attending the school in grades $8-11$. The majority (86.8\%) of the teachers also considered the school to be an appropriate setting for this, although only half $(50 \%, n=19)$ said that they would be willing to participate in such a programme, and even less $(47.4 \%, n=18)$ were willing to sacrifice class time to facilitate such a programme. Only $34.2 \%(n=13)$ of the teachers who participated in the study answered yes to all the preceding questions. No significant differences between the male and female teachers were found with regard to these variables.

Neither gender, age, total years teaching, nor years spent teaching at a specific school, were found to significantly predict the answers to questions relating to attitudes. However, this may relate to the small sample size and wide confidence intervals.

No significant relationship was found between the size of either the teachers' estimates or accuracy with regard to disordered eating attitudes, and their answers to questions on the need for a preventative programme to address disordered eating attitudes, their perception of the school as an appropriate place in which to address disordered eating attitudes, and their willingness to both participate and sacrifice class time to do this. This may once again relate to the small sample size and large confidence intervals observed.

\section{Discussion}

This is the first published study to document the presence of abnormal eating attitudes in South African Jewish adolescent girls, and first to record the willingness of teachers to participate in preventative programmes in this regard. Upon consideration of the results gathered by the EAT-26, both the mean score (12.1) and the subjects scoring $\geq 20(20 \%)$ fell within the upper range of the spectrum of results of previous studies ${ }^{12,14,15,19,22,26}$ conducted on a local and international scale.

The mean EAT-26 score and the percentage of adolescent girls scoring $\geq 20$ in this study was higher than the rates reported by the National Eating Disorders Screening Program (a mean of 9.5 and a prevalence of $14.7 \%$ for white adolescent girls) which coordinated the first-ever nationwide eating disorder screening initiative for high schools in the USA. ${ }^{29}$ However, this should be interpreted with caution, as definitions of disordered eating behaviour differ between studies.

Table III compares the setting and results of the current study to four South African studies previously conducted using the EAT-26, although methodological issues make direct comparisons difficult. 
Table III: Comparison of the current study with four studies previously conducted in South Africa

\begin{tabular}{|c|c|c|c|c|c|}
\hline Author and date & Gurrent study & Szabo and Hollands ${ }^{12}$ & Cadaras et al ${ }^{15}$ & Szabo and Allwood ${ }^{14}$ & Le Grange et al26 \\
\hline Setting & $\begin{array}{l}\text { Co-educational } \\
\text { Jewish high school in } \\
\text { Johannesburg }\end{array}$ & $\begin{array}{l}\text { Racially diverse private } \\
\text { school in Johannesburg }\end{array}$ & $\begin{array}{l}\text { Five state-owned } \\
\text { secondary schools in the } \\
\text { greater Cape Town area }\end{array}$ & $\begin{array}{l}\text { Three multiracial, } \\
\text { girls-only schools in } \\
\text { Johannesburg }\end{array}$ & $\begin{array}{l}\text { Male and female students } \\
\text { at four South African high } \\
\text { schools and undergraduate } \\
\text { social science students in } \\
\text { Cape Town }\end{array}$ \\
\hline Response rates & $85 \%$ & $76 \%$ & $88 \%$ & $86 \%$ & Almost $100 \%$ \\
\hline Sample size & 220 & 213 & 228 & 1353 & 895 \\
\hline Gender & Female & Female & Female & Female & Male and female \\
\hline Grades & $8-11$ & $8-12$ & $10-12$ & Not quoted & Not quoted \\
\hline Mean age & 15.68 (SD 1.2) & 14.7 (SD 1.4) & Not quoted & Not quoted & $\begin{array}{l}17.2 \text { (SD 1.7) (male and } \\
\text { female) } \\
\text { Adolescent subset: } 16.3 \text { (SD } \\
0.7 \text { ) (male and female) }\end{array}$ \\
\hline $\begin{array}{l}\text { Mean EAT-26 } \\
\text { score }\end{array}$ & Total: 12.1 (SD 11.2) & $\begin{array}{l}\text { Total: } 11.7 \text { (SD 11.4) } \\
\text { White: } 11.5 \text { (SD 11.6) } \\
\text { Black: } 16.2 \text { (SD 10.5) }\end{array}$ & $\begin{array}{l}\text { Total: } 12.2 \text { (SD 12.6) } \\
\text { White: } 12.5 \text { (SD 11.6) } \\
\text { Black: } 11.7 \text { (SD 10.8) } \\
\text { Mixed race: } 11.5 \text { (SD } \\
\text { 11.2) }\end{array}$ & $\begin{array}{l}\text { Total: Not quoted } \\
\text { White: } 12.3 \text { (SD 10.2) } \\
\text { Black: } 12.5 \text { (SD 9) }\end{array}$ & $\begin{array}{l}\text { Total: Not quoted } \\
\text { White female adolescents: } \\
13.8 \text { (SD 10.8) } \\
\text { Black female adolescents: } \\
12.3 \text { (SD 7.7) } \\
\text { Male race female } \\
\text { adolescents: } 9 \text { (SD 7.9) }\end{array}$ \\
\hline$\%$ with EAT $\geq 20$ & $20 \%$ & $\begin{array}{l}\text { Total: } 21.6 \% \\
\text { White: } 20.7 \% \\
\text { Black: } 37.5 \%\end{array}$ & $\begin{array}{l}\text { Total: } 18.8 \% \\
\text { White: } 21.2 \% \\
\text { Black: } 17.9 \% \\
\text { Mixed race: } 17.1 \%\end{array}$ & $\begin{array}{l}\text { Total: Not quoted } \\
\text { White: } 18.6 \% \\
\text { Black: } 18.7 \%\end{array}$ & $\begin{array}{l}\text { Total: } 14 \% \\
\text { White: Not quoted } \\
\text { Black: Not quoted }\end{array}$ \\
\hline $\begin{array}{l}\text { Bulimia subscale } \\
\text { (mean) }\end{array}$ & $2.4(2.8)$ & Not quoted & Not quoted & $\begin{array}{l}\text { White: } 1.3(2.6) \\
\text { Black: } 1.6(2.4)\end{array}$ & Not quoted \\
\hline $\begin{array}{l}\text { Dieting subscale } \\
\text { (mean) }\end{array}$ & $7.9(8.5)$ & Not quoted & Not quoted & $\begin{array}{l}\text { White: } 8.5 \text { (7.9) } \\
\text { Black: } 7.7(6.8)\end{array}$ & Not quoted \\
\hline $\begin{array}{l}\text { Oral control } \\
\text { subscale (mean) }\end{array}$ & $1.9(2.2)$ & Not quoted & Not quoted & $\begin{array}{l}\text { White: } 3.8 \text { (5.8) } \\
\text { Black: } 3.2(3)\end{array}$ & Not quoted \\
\hline
\end{tabular}

EAT-26: Eating Attitudes Test (26-item version), SD: standard deviation

The study by Le Grange et $\mathrm{al}^{26}$ differed from the other studies in that the sample included both male and female participants. Therefore, it is not surprising that the proportion of subjects in this group who scored at or above the cut-off of 20 on the EAT-26 was the lowest recorded $(14 \%)$, given that adolescent girls are more commonly affected by eating disorders. ${ }^{1}$ In the study by Szabo and Hollands, ${ }^{12}$ the mean EAT-26 score increased with advancing grades, compared to the current study which found that the mean EAT-26 score was associated with the grade, but not the age. In view of the fact that the association between the grade and mean EAT-26 score was of borderline significance, and that the grade was not found to be predictive of the EAT-26 score when entered into a regression, conclusions regarding this finding will not be drawn.

A comparison of the current study with two other studies using the EAT-26 on Jewish adolescents (Table IV) shows that Jewish adolescent girls in the Canadian study by Pinhas et $\mathrm{al}^{19}$ scored significantly higher on the EAT-26 than their non-Jewish counterparts (Jewish mean 13.5, SD 8.8 versus non-Jewish mean 11.9, SD 8.3). When comparing our results to those of white adolescent females in the South African studies cited previously, this effect was not noted. However, it is important to bear in mind that our study did not include a non-Jewish comparison group.
Thus, a direct comparison of this study and the other South African studies might not yield accurate results owing to subtle differences in the execution thereof. The results of the current study are similar to those of an Israeli study 22 in which a prevalence of $19.5 \%$ of EAT26 scores at or above threshold was reported.

An examination of the scores for individual items of the EAT-26 revealed high rates of eating and weight-related concerns, even if subjects did not surpass the cut-off point of 20. Additionally, it was indicated that exercise in this group was more often than not performed with at least some focus on its potential to promote weight loss, needed to detect early and concealed weight-loss attempts in normal-weight adolescents.

It is to be expected that subjects with behavioural manifestations of disordered eating are identified by the EAT-26 as having a potential eating disorder. Thus, it was not surprising that engagement in extreme weight-loss behaviour over the past year was found to be a significant predictor of the total EAT-26 score and of an EAT-26 score $\geq 20$. The finding that regarding oneself as being overweight predicted the total score and a score $\geq 20$, was in keeping with previous reports that a perception of being overweight correlated with eating problems in adolescence, ${ }^{30}$ as well as with a greater likelihood of engaging in weight-loss behaviour. ${ }^{31}$ 
Table IV: Comparison of the current study with two other studies which used the 26-item version Eating Attitudes Test on Jewish adolescents

\begin{tabular}{|c|c|c|c|}
\hline & Current study & Latzer and Tzischinsky'22 & Pinhas et al ${ }^{19}$ \\
\hline Year & $2010 / 2011$ & 2005 & 2008 \\
\hline Country & South Africa & Israel & Canada \\
\hline Setting & Traditional school in urban area & $\begin{array}{l}\text { Five different middle and high schools in } \\
\text { five different residential areas }\end{array}$ & Toronto high school students \\
\hline Gender & Female & Female & Male and female \\
\hline Response rate & $84 \%$ & Not reported & $82 \%$ \\
\hline Participant numbers & 220 & 1270 & $\begin{array}{l}\text { Total: } 1868 \\
\text { Male: } 1145 \\
\text { Female: } 1130\end{array}$ \\
\hline Written consent from parents & Yes & Not reported & Not reported \\
\hline Grades & $8-11$ & Not reported & Not reported \\
\hline Age range & $13-19$ (mean 15.68) & $12-18$ & $13-20$ \\
\hline Mean EAT-26 score & $12.3(11.2)$ & $\begin{array}{l}\text { For different groups ranging from } \\
10.2(7.8) \text { to } 15.9(10.2)\end{array}$ & $\begin{array}{l}\text { Jewish females: } 13.5 \text { (8.8) } \\
\text { Non-Jewish females: } 11.9 \text { (8.3) } \\
\text { Jewish males: } 8.9(8.7) \\
\text { Non-Jewish males: } 9.1(8.4)\end{array}$ \\
\hline$\%$ with EAT-26 $\geq 20$ & $20 \%$ & $19.5 \%$ & $\begin{array}{l}\text { Jewish females: } 25 \% \\
\text { Non-Jewish females: } 18 \% \\
\text { Jewish males::Not reported } \\
\text { Non-Jewish males: Not reported }\end{array}$ \\
\hline Bulimia subscale (mean) & $2.4(2.8)$ & 1 (1) to $3.4(3.1)$ & $\begin{array}{l}\text { Jewish females: } 2.1(2.6) \\
\text { Non-Jewish females: } 1.6(2.5) \\
\text { Jewish males: } 1.8(0.1) \\
\text { Non-Jewish males: } 1.7(0.1)\end{array}$ \\
\hline Dieting subscale (mean) & $7.9(8.5)$ & $\begin{array}{l}\text { For different groups ranging from } 6.3 \\
(6.3) \text { to } 8.2(6)\end{array}$ & $\begin{array}{l}\text { Jewish females: } 8.5 \text { (6.1) } \\
\text { Non-Jewish females: } 6.6 \text { (5.1) } \\
\text { Jewish males: } 4.4 \text { (5.2) } \\
\text { Non-Jewish males: } 4.1 \text { (5) }\end{array}$ \\
\hline Oral control subscale (mean) & $1.9(2.2)$ & $3(2.7)$ to $4.1(3.1)$ & $\begin{array}{l}\text { Jewish females: } 3.5(3.4) \\
\text { Non-Jewish females: } 3.9(3.2) \\
\text { Jewish males: } 3.1(0.2) \\
\text { Non-Jewish males: } 3.5(0.2)\end{array}$ \\
\hline
\end{tabular}

EAT-26: Eating Attitudes Test (26-item version)

Adolescents who had dieted in the preceding year were more than three times more likely to score $>20$. This phenomenon was shown in longitudinal studies that implicated dieting in the development of eating disorders. ${ }^{32}$ It also provides support for the suggestion that dieting in adolescence should be regarded as risk-taking behaviour. ${ }^{16}$ Adolescent girls who had dieted in order to actively lose weight were even more likely to have scores $\geq 20$. Girls who believed that they were overweight and who had dieted in the past year were more than four times more likely to have EAT-26 scores $\geq 20$ than those with one or both negative.

The above findings are relevant to the early detection of adolescent girls at risk of developing an eating disorder. Both parents and teachers should be made aware that girls who engage in extreme methods of weight control require attention. Similarly, those who express concern that they are overweight or have used dieting as a means of gaining weight control in the past year should also be carefully observed for the development of more severe symptoms.

In the current study, almost two thirds (64\%) of the adolescents reported trying to lose weight at the time of the study, and more than two thirds $(67.3 \%)$ had used dieting as a means of gaining weight control in the preceding year. These results are in keeping with prior reports that $33-66 \%$ of teenage girls are dieting at any given point in time. ${ }^{33,34}$ The findings of prior reports that most adolescent dieters are of a normal weight ${ }^{35}$ were in keeping with the finding in this study that just over half $(53.3 \%)$ of the adolescents trying to lose weight at the time of the study regarded themselves as being of the right weight. It is conceivable in the context of Western culture which promotes a "thin ideal", that some learners who regarded themselves as being overweight were actually within normal anthropometrical ranges. Thus, it is possible that an even greater percentage of adolescent girls with an objectively measured normal weight were attempting weight loss than that estimated by the study. This is concerning, given that dieters of normal body weight have been found to be eight times more likely to develop an eating disorder than their non-dieting counterparts. ${ }^{36}$

It has been noted that women who have developed cognitive schemas that associate being underweight with positive attributes report higher eating disorder symptoms. ${ }^{37}$ In this study, being underweight rather than the correct weight appeared to be viewed by many subjects as desirable. This was suggested by the fact that $56.6 \%$ of the adolescents who regarded themselves as being the correct 
weight were trying to lose weight. Only a small proportion (7.7\%) of girls who regarded themselves as being underweight was trying to gain weight, while most were satisfied to remain at this weight by either doing nothing about their weight or by actively trying to maintain it. $15.4 \%$ of the adolescents who considered themselves to be underweight were actively attempting to lose weight. Society and the media promote "ultra-thinness" as an ideal or desirable trait, and this may have been internalised by many teenage girls at this particular school. This echoes the observation of a school effect ${ }^{15}$ with regard to eating attitudes and body shape concerns, according to which it is hypothesised that the existence of a school culture may create an environment in which peer influences potentiate or protect against the development of eating pathology. ${ }^{15}$ Thus, a shift with regard to body shape preference may be required.

As expected, the percentage of adolescent girls trying to lose weight was higher in the group which considered itself to be slightly overweight $(87.7 \%)$ than that in the group which believed itself to be the correct weight $(56.6 \%)$. However, it was surprising that a smaller proportion $(71.4 \%)$ of the adolescents who regarded themselves as being "very overweight" were trying to lose weight than that pertaining to the adolescents in the group who believed itself to be slightly overweight. It is conceivable that some of the girls who consider themselves to be very overweight may despair of achieving the "thin ideal" espoused by the culture that is prevalent in their environment and therefore may not even attempt weight reduction.

Almost one in three adolescent girls in the school (30.2\%) were found to require evaluation for a potential eating disorder based on the EAT-26 scores and the presence of extreme weight-loss behaviour. Of these, only $9.2 \%$ reported having ever being diagnosed with an eating disorder. These results are in line with the USA National Eating Disorders screening programme, which found that $83-86 \%$ of girls with symptoms of abnormal eating had not received treatment. ${ }^{29}$ These findings are of relevance as it has been shown that a shorter period between symptom onset and treatment commencement may improve prognosis with regard to recovery in both anorexia nervosa and bulimia nervosa. ${ }^{38}$ Therefore, detection and early referral for treatment of at-risk individuals should be prioritised.

It is important to consider that a comparison of the mean of the teachers' estimates (predictions of learner reponses) and that of the questionnaire rate may be affected because of underestimation of the true prevalence rate indicated by the questionnaire. This could result from either the response bias of the learners or underreporting, a known limitation of the EAT-26 instrument. ${ }^{39}$ Therefore, it is important to note that the difference between teacher and learner responses should not be regarded as a direct indicator of teachers' accuracy. In cases where teachers' estimates exceeded the calculated results for learners, it is possible that this effect would have been reduced or even reversed if bias or under-reporting were eliminated. By contrast, where teachers' estimates were below the calculated prevalence rate, this would actually be more marked if the effects of bias or under-reporting were eliminated. This is likely to represent a true underestimation of the situation by the teachers, and is indicative of the fact that the teachers were not fully aware of the extent of weight-loss behaviour at the school. They were also not fully cognisant of how widespread disordered eating attitudes and behaviour was at the school, and the need for referral to appropriate healthcare professionals.

Teachers who were female, younger and of the Jewish faith and who had a shorter duration of total teaching time, predicted higher estimates for some of the variables under investigation. If one considers that the learners' arm of the study may have underrepresented the prevalence rates, these teachers' estimates may be more reflective of the reality of the situation than those of the other teachers. This is plausible as teachers in this group could be representative of those most likely to be affected by eating-related issues themselves, although this is a tentative interpretation. The majority of the teachers in the current study acknowledged the need to address and/or prevent abnormal eating attitudes and/or behaviour in the adolescents attending the school. The school was also regarded by the vast majority of teachers as an appropriate place in which this could occur, although only half of them were willing to participate in such a programme, and even fewer prepared to sacrifice class time. These findings echo the observation made by McVey et al that the engagement of teachers in their study was not optimal. ${ }^{40}$ The findings also suggest that although the teachers displayed awareness of the problem and the necessary required action, this didn't appear to be sufficient to obtain their cooperation.

In terms of the limitations of the study, it cannot be assumed that these findings can be generalised to other South African traditional Jewish schools. The overall prevalence rate of abnormal eating attitudes may have been affected by the exclusion of the grade $12 \mathrm{~s}$, as well as response bias and under-reporting owing to the nature of the questionnaire. The prevalence rate for eating disturbances meeting the criteria for an eating disorder diagnosis could not be determined. Logistical issues, as well as the emotional consequences of performing anthropometrical measurements in this particular setting, resulted in actual weight and height not being taken. As the study was cross-sectional in nature, causal inferences regarding the direction of the associations could not be made. Furthermore, a lower response rate resulted in a smaller sample size of teachers being included in the study, which may have affected the statistical analysis. Respondent bias may be a factor as it is conceivable that teachers who participated were more aware of and concerned about the topic of the study than those who did not. A quantitative approach is often a first step, but is not always ideal with regard to obtaining in-depth insight into teachers' attitudes towards eating disorders. Hence, a qualitative exploration of this in future could yield further valuable insight.

\section{Conclusion}

This study documents the presence of abnormal eating attitudes and weight-loss behaviour in South African Jewish female adolescents. The prevalence of abnormal eating attitudes, measured by the EAT26 , fell within the upper range of that reported by similar studies, conducted both nationally and internationally. Our findings suggest that any given school may be governed by a culture, according to which the "ultra-thin" ideal is valued. Further quantitative and qualitative exploration of this phenomenon is needed to corroborate 


\section{this suggestion.}

Given the high prevalence of eating disorders ${ }^{4}$ and their vast impact on the morbidity and mortality of young women, ${ }^{4}$ as well as difficulties managing them, ${ }^{1}$ the findings of the current study, together with other similar South African studies, suggest that there is a need for programmes that address abnormal eating attitudes and behaviour in South African schools. The success of such programmes would largely be dependent upon an ecological-type approach, which includes the participation of learners, their parents and teachers in a school-based environment. ${ }^{41-43}$ Additional research is necessary to investigate the attitudes of parents and learners to such school programmes. Further investigation of teacher attitudes is also warranted, given the preliminary findings of the current study. Such research could yield valuable information which may facilitate greater cooperation from all viable role players in the school community.

\section{Acknowledgements}

The authors thank Ms Lauren Philips for editorial assistance with the drafting of the manuscript.

\section{References}

1. Fairburn CG, Harrison PJ. Eating disorders. Lancet 2003;361(9355):407-416.

2. McCarthy M. The thin ideal, depression and eating disorders in women. Behav Res Ther. 1990;28(3):205-215

3. Kaplan H, Sadock B. Kaplan and Sadock's synoposis of psychiatry. $8^{\text {th }}$ ed. London: Lippincott Williams \& Wilkins; 1998.

4. World Health Organization. Prevention of mental disorders: effective interventions and policy options [homepage on the Internet]. 2004. c2014. Available from: http://apps. who. int/iris/bitstream/10665/43027/1/924159215X_eng.pdf

5. American Psychiatric Association. Diagnostic and statistical manual of mental disorders. 4th ed. Washington: American Psychiatric Association; 1994.

6. Nasser M. Culture and weight consciousness. J Psychosom Res. 1988;32(6):573-577.

7. Puoane T, Fourie JM, Shapiro M, et al. "Big is beautiful": an exploration with urban black community health workers in a South African township. South Afr J Clin Nutr. 2005;18(1):6-15.

8. Hawkins N, Richards PS, Granley HM, Stein DM. The impact of exposure to the thin-ideal media image on women. Eat Disord. 2004;12(1):35-50.

9. Garret CJ. Recovery from anorexia nervosa: a Durkheimian interpretation. Soc Sci Med 1996;43(10):1489-1506.

10. Mitchel JE, Erlander M, Pyle RL, Fletcher LA. Eating disorders, religious practices and pastoral counselling. Int J Eat Disord. 1990;9:589-593.

11. Beumont PJ, George GCW, Smart DE. "Dieters" and "vomiters" and "purgers" in anorexia nervosa. Psychol Med. 1976;6(4):617-632.

12. Szabo CP, Hollands C. Abnormal eating attitudes in secondary-school girls in South Africa: a preliminary study. S Afr Med J. 1997;87(4 Suppl):524-526.

13. Szabo CP, Berk M, Tlou E. Eating disorders in black female South Africans. A series of cases. S Afr Med J. 1995;85:588-590.

14. Szabo CP, Allwood CW. A cross-cultural study of eating attitudes in adolescent South African females. World Psychiatry. 2004;3(1):41-44.

15. Cadaras AA, Lambert EV, Charlton E. An ethnic comparison of eating attitudes and associated body image concerns in adolescent South African schoolgirls. J Hum Nutr Diet. 2001;14(2):111-120

16. Szabo CP. Youth at risk - dieting and eating disorders: a South African Perspecive. S Afr Med J. 2002;92(4):282-283

17. Skyes DK, Leuser B, Melia M, Gross M. A demographic analysis of 252 patients with anorexia nervosa and bulimia. Int J Psychosom. 1988;35(1-4):5-9.

18. Kim KH. Religion, weight perception and weight control behaviour. Eat Behav. 2007;8(1):121-131

19. Pinhas L, Heinman M, Bryden P, et al. Disordered eating in Jewish adolescent girls. Can
J Psychiatry. 2008;53(9):601-608.

20. Feinstein MC, Meir A. Disordered eating and religious observance: a focus on ultraOrthodox Jews in an adult community study. Int J Eat Disord. 2012;45(1):101-109.

21. Gluck ME, Geliebter A. Body image and eating behaviours in Orthodox and secular Jewish women. J Gend Specific Med. 2002;5(1):19-24.

22. Latzer $Y$, Tzischinsky 0 . Eating attitudes in a diverse sample of Israeli adolescent females: a comparison study. J Adolesc. 2005;28(3):317-323.

23. Latzer $\mathrm{Y}$, Tzischinsky 0 , Gefen $\mathrm{S}$. Level of religiosity and disordered eating psychopathology among modern-orthodox Jewish adolescent girls in Israel. Int $\mathrm{J}$ Adolesc Med Health. 2007;19(4):511-512.

24. Garner DM, Olmsted MP, Bohr Y, Garfinkel PE. The Eating Attitudes Test: psychometric features and clinical correlates. Psychol Med. 1982;12(4):871-878.

25. 2009 state and local Youth Risk Behavior Survey. Centers for Disease Control and Prevention [homepage on the Internet]. 2009. c2014. Available from: http://www.cdc. gov/healthyyouth/yrbs/pdf/questionnaire/2009_hs_questionnaire.pdf

26. Le Grange D, Louw J, Russell B, et al. Eating attitudes and behaviours in South African adolescents and young adults. Transcult Psychiatry. 2006;43(3):401-417.

27. Szabo CP, Allwood CW. Application of the Eating Attitudes Test (EAT-26) in a rural, Zulu speaking, adolescent population in South Africa. World Psychiatry. 2004;3(3):169-171.

28. Top five Youth Risk Behavior Surveillance System (YRBSS) frequently asked questions. Centers for Disease Control and Prevention [homepage on the Internet]. 2009. c2014. Available from: http://www.cdc.gov/healthyyouth/yrbs/faq.htm\#conducting

29. Austin SB, Ziyadeh NJ, Forman S, et al. Screening high school students for eating disorders: results of a national initiative. Prev Chronic Dis [homepage on the Internet]. 2008. c2014. Available from: http://www.cdc.gov/pcd/issues/2008/oct/07_0164.htm

30. Toro J, Castro J, Garcia M, et al. Eating attitudes, sociodemographic and body shape evaluation in adolescence. Br J Med Psychol. 1989;62(Pt 1):61-70.

31. Chung AE, Perrin EM, Skinner AC. Accuracy of child and adolescent weight perceptions and their relationships to dieting and exercise behaviours: a NHANES Study. Acad Pediatr. 2013;13(4):371-378

32. Hsu LKG. Can dieting cause an eating disorder? Psychol Med. 1997; 27: 509-513.

33. Schleimer K. Dieting in teenage schoolgirls. A longitudinal prospective study. Acta Paediatr Scand Suppl. 1983;312:1-54.

34. Heuneman RL, Shapiro LR, Hampton MC, Mitchell BW. A longitudinal study of gross body composition and body conformation and their association with food and activity in a teenage population. Am J Clin Nutr. 1996;18(5):325-388.

35. Patton GC, Carlin JB, Shao Q, et al. Adolescent dieting: health weight control or borderline eating disorder? J Child Psychol Psychiatry. 1997;38(3):299-306.

36. Patton GC, Johson-Sabine E, Wood K, et al. Abnormal eating attitudes in London schoolgirls - a prospective epidemiological study: outcome at 12 month follow-up. Psychol Med. 1990;20(2):383-394

37. Ahern AL, Bennet KM, Hetherington MM. Internalization of the ultra-thin ideal: positive implicit associations with underweight fashion models are associated with drive for thinness in young women. Eat Disord. 2008;16(4):294-307.

38. Reas DS, Williamson DA, Martin DC, Zucker NL. Duration of illness predicts outcome for bulimia nervosa: a long-term follow-up study: Int J Eat Disord. 2000;27(4):428-434.

39. Garner DM, Garfunkel PE. The Eating Attitudes Test: an index of the symptoms of anorexia nervosa. Psychol Med. 1979;9(2):273-327.

40. McVey G, Tweed S, Blackmore E. Healthy schools - healthy kids: a controlled evaluation of a comprehensive universal eating disorder prevention program. Body Image. 2007;4(2):115-136.

41. Neumark-Sztainer DR, Wall MM, Naines JL, et al. Shared risk and protective factors for overweight and disordered eating in adolescents. Am J Prev Med. 2007;33(5):359-369.

42. Wilksch SM, Tiggemann M, Wade TD. Impact of interactive school-based media literacy lessons for reducing internalization of media ideals in young adolescent girls and boys. Int J Eat Disord. 2006;39(5):385-393.

43. Russell-Mayhew S, Arthur N, Ewashen C. Targeting students, teachers and parents in a wellness-based prevention program in schools. Eat Disord. 2007;15(2):159-181. 